\title{
Management of Portal/Mesenteric Vein Thrombosis
}

\author{
Jörg C. Kalffa Robert Thimme ${ }^{\text {b }}$ \\ a Department of Surgery, University Hospital Bonn, Bonn, Germany, \\ ${ }^{b}$ Department of Medicine II, University Hospital Freiburg, Freiburg i.Br., Germany
}

Portal vein thrombosis (PVT)/mesenteric vein thrombosis (MVT) is a rare but clinically relevant and important vascular disorder. The underlying causes of PVT/MVT are often multifactorial and include inherited thrombophilias, malignancies, liver cirrhosis, infections, or other processes localized to the epigastrium and hepatobiliary system. The three main categorical groups include malignant, cirrhotic, and non-malignant, non-cirrhotic PVT. Not only the etiology but also the site of thrombosis, its extension, and the natural course (progressive or self-resolving) determine the clinical presentation, the complications, and also the available treatment algorithms. Despite its high clinical relevance and possible deleterious clinical course, little information is currently available about optimal therapy strategies, and guidelines are not available. For this special issue, we have chosen a group of leading experts regarding the diagnosis and treatment of PVT/MVT to give important insights into its etiology, diagnosis, complications, and therapeutic options. Due to the fact that randomized studies including large patient cohorts are not available, we have selected experts from different specialties to state their point of view based on the literature as well as personal experience. Trebicka and Strassburg [1] summarize current concepts on etiologies, risk factors, and complications of this heterogeneous condition in adults. The diagnosis is based on imaging. The value and relevance of different methods, such as ultrasound, computed tomography, and magnetic resonance imaging, are summarized by Hauenstein and Li [2]. Nonsurgical therapeutic options in PVT may differ and have to consider the etiology, e.g. the presence of infection or tumors. The according therapy algorithms are summarized by
Schultheiss et al. [3]. The article by Lang et al. [4] points out the interdisciplinary approach that is required to select an optimal individual therapy for a given patient based on clinical presentation, underlying diseases, extension of thrombosis, and the patient's comorbidities. A particular focus is therefore put on the surgical therapeutic options. A specific clinical challenge is a PVT in patients with liver cirrhosis. Rössle et al. [5] suggest therapy algorithms favoring TIPS (transjugular intrahepatic portosystemic shunt) as a first-line treatment for these patients. These algorithms are based on studies published in the last 10 years and on the clinical experiences of the authors. Portal hypertension is a major complication of PVT. Thus, in a final review article, Glowka et al. [6] summarize the clinical management of chronic portal hypertension with a specific focus on surgical therapeutic options. Finally, clinically relevant but also controversial aspects in the management of PVT are highlighted in the discussion chaired by Sauerbruch (with the participants Hopt, Neeff, Pötzsch, Rössle, and Valla) [7].

In sum, we hope that these articles written by leading experts in the field of PVT/MVT will guide you in the diagnosis and therapy of patients with PVT/MVT. A central message of all articles is the necessity of a multidisciplinary approach. Clearly, additional prospective studies are needed to confirm the therapy algorithms suggested by the experts in the near future. We sincerely hope that you consider these combined and comprehensive reviews and discussions as important for your own clinical experience and would like to thank all experts for their excellent contributions.

\section{KARGER \\ Fax +497614520714




\section{References}

1 Trebicka J, Strassburg CP: Etiology and complications of portal vein thrombosis. Viszeralmedizin 2014;30:375-380.

2 Hauenstein K, Li Y: Radiological diagnosis of por$\mathrm{tal} /$ mesenteric vein occlusion. Viszeralmedizin 2014;30:382-387.

3 Schultheiß M, Bettinger D, Thimme R: Nonsurgical therapeutic options in portal vein thrombosis Viszeralmedizin 2014;30:388-392.
4 Lang SA, Loss M, Wohlgemuth WA, Schlitt HJ: Clinical management of acute portal/mesenteric vein thrombosis. Viszeralmedizin 2014;30:394-400.

5 Rössle M, Bausch B, Klinger C: Therapy algorithm for portal vein thrombosis in liver cirrhosis: the internist's point of view. Viszeralmedizin 2014;30: 401-408.
6 Glowka TR, Kalff JC, Schäfer N: Clinical management of chronic portal/mesenteric vein thrombosis: the surgeon's point of view. Viszeralmedizin 2014; 30:409-415.

7 Sauerbruch T: Management of portal $/$ mesenteric vein occlusion (Interdisciplinary Discussion). Viszeralmedizin 2014;30:417-420. 